\title{
Special issue on emerging trends, challenges and applications in cloud computing
}

\author{
Longzhi Yang ${ }^{1} \cdot$ Vijayakumar Varadarajan ${ }^{2} \cdot$ Tossapon Boongoen $^{3} \cdot$ Nitin Naik $^{4}$
}

Published online: 23 December 2021

(C) The Author(s), under exclusive licence to Springer Science+Business Media, LLC, part of Springer Nature 2021

Cloud computing enables ubiquitous and efficient on-demand access to information, data, and computational resources with the support of modern wired and wireless communication technologies. Cloud computing has been very widely used in education, autonomous vehicles, smart cities/homes, renewable energy, healthcare, engineering, business, and telecommunications, amongst others, with the support of the advances in Artificial Intelligence, Internet of Things (IoT), and Data Science. Such technologies and their applications have made significant impact to the way people live and do business by offering online services and instant communications.

Despite of the intensive research effort in the field, some open challenges remain, such as inefficient load balancing and energy management in cloud data centres, high cost to access to the facility, insufficient security and privacy, and low speed big data stream processing. This special issue focuses on recent advances in addressing these challenges and investigating all the aspects of emerging trends, in addition to reporting innovative real-world applications of cloud computing to deliver effective and efficient solutions

Longzhi Yang

longzhi.yang@northumbria.ac.uk

Vijayakumar Varadarajan

v.varadarajan@unsw.edu.au

Tossapon Boongoen

tossapon.boo@mfu.ac.th

Nitin Naik

n.naik1@ aston.ac.uk

1 Department of Computer and Information Sciences, Northumbria University, Newcastle upon Tyne, UK

2 School of Computer Science and Engineering, The University of New South Wales, Sydney, Australia

3 School of Information Technology, Mae Fah Luang University, Chiang Rai, Thailand

4 School of Informatics and Digital Engineering, Aston University, Birmingham, UK for current and future challenges. These papers have been selected after a rigorous peer review process.

Cloud computing and wireless technologies provide the technological foundation for IoT, in terms of data, computational, and communication solutions. The first paper in this special issue introduces an open source IoT-based smart home management system using sensors, actuators, smartphones, web services and micro-controllers [1]. The system has been prototyped and deployed in a bedroom for system validation and evaluation. Compared to the approaches available in the literature, the proposed system is featured by better performance in terms of cost efficiency, open source, energy efficiency, reliability, and scalability.

Security, privacy, and trust are important aspects to consider when we use cloud computing services, and image processing is a common task for cloud computing. A common solution from many cloud services for trusted and secure image classification task is using encryption, but encryption can be useless in preserving privacy in many cases in an adversarial setting. The second paper therefore proposes a cloud-based differentially private image classification approach that protects the privacy of individuals who appeared in an image dataset [2]. The performance of the proposed framework is validated by considering the trade-off between the accuracy and privacy, with promising results demonstrated.

Blockchain has emerged as a key technology for secure, privacy-preserving, and trustworthy digital systems, and many innovative applications have been developed in the recent years after its success in digital currency. Electronic government (E-government) is one of the most complex systems which needs to be distributed, secured and privacy-preserved, and the blockchain technology appears as a natural solution for this. The next paper proposes a framework of a decentralised e-government system using the blockchain technology, ensuring information security, privacy, and trust [3]. Theoretical and quantitative analysis 
demonstrates the implications of the proposed framework in public sectors.

Fog computing extends could computing by an extra fog layer between the end devices and the cloud, leading to low bandwidth and energy consumption, low latency, higher location awareness, and higher quality of services. Such technologies have been widely applied in the areas of autonomous vehicles, healthcare, smart grid, cloud gaming amongst others. It is important to keep such systems secure due to the importance of privacy in the society and the presence of very strong cyber-attack threats. The next article presents an identity-based and anonymous key agreement protocol for fog computing [4]. Based on this scheme, the communication between the sensor devices and the fog devices are not traceable by the central servers, which is implemented with limited computational and communication overhead.

The on-demand usage nature of cloud computing may affect the availability and reliability of services in the cloud, as the demand may peak beyond the maximum capacity of the cloud resources. Resource optimisation is one of the common approaches to address this, especially with the emergence of Software Defined Networking (SDN). The next publication in this special issue proposes a resource optimisation method for SDN enabled big data stream processing in a could environment [5]. The system was simulated in the CloudSimSDN platform, showing the working mechanism and the usefulness of the method.

Load balancing is another approach that has been very widely implemented in cloud for effective resource management and allocation to meet the dynamic on-demand resource request. The next article reports a new load balancing technique using double Q-learning for workload distribution between virtual servers in a cloud environment [6]. This approach effectively ensures a higher quality of service of the cloud especially when there are a large number of users. This technique can optimise resource allocation and thus improve consumer satisfaction.

Cloud computing is often an integrity part of modern business solutions, in the field of education, entertainment, health care, business and finance, amongst others, and could computing has become a main platform for business intelligence. The next paper reports an investigation of the application of business intelligence in the higher education sector using a case study [7]. It is suggested from this study that the integration of business intelligence to academic processes can provide meaningful insights and decision support to many challenges.

An effective electrical distribution network with the support of an efficient battery-base energy storage system is essential for a nation's development and net-zero carbon goal. The next paper presents a supervision strategy in an Arduino card photovoltaic generator with storage connected to the low voltage grid, to address the overload and underload problems of batteries [8]. This is implemented by integrating supervision algorithms to the Arduino card, and the simulation results demonstrate the effectiveness of the system for battery protection and system supervision.

Given the wide range of options available in the cloud computing market, it is appealing to study the strengths and weaknesses of the tariffs form various cloud computing providers. The next article in this special issue reports a new costing model that systematically evaluates cloud services by taking multiple resourcing factors into consideration [9]. The proposed work was validated and evaluated using a real-world dataset extracted from a data centre. This comparative study suggests that could computing cost may vary up to $17 \%$ from different cloud service providers.

Data centres are important building blocks for cloud computing services, which are one of the major contributors in the computing sector towards carbon emissions. Along with the increasing demand of cloud services, energy efficiency of data centres has become a key aspect for greenhouse gas reduction. The next paper presents a predictive model of energy consumption estimation for servers in cloud centres [10]. The experimental results show that the server fabrication, ambient temperature, and CPU temperature have noticeable effects on the power consumption of servers.

\section{References}

1. Naik, K., \& Patel, S. (2018). An open source smart home management system based on IOT. Wireless Networks. https://doi. org/10.1007/s11276-018-1884-z

2. Chicha, E., Al Bouna, B., Nassar, M., et al. (2018). Cloud-based differentially private image classification. Wireless Networks. https://doi.org/10.1007/s11276-018-1885-y

3. Elisa, N., Yang, L., Chao, F., et al. (2018). A framework of blockchain-based secure and privacy-preserving E-government system. Wireless Networks. https://doi.org/10.1007/s11276-0181883-0

4. Patonico, S., Braeken, A., \& Steenhaut, K. (2019). Identity-based and anonymous key agreement protocol for fog computing resistant in the Canetti-Krawczyk security model. Wireless Networks. https://doi.org/10.1007/s11276-019-02084-6

5. Al-Mansoori, A., Abawajy, J., \& Chowdhury, M. (2018). SDN enabled BDSP in public cloud for resource optimization. Wireless Networks. https://doi.org/10.1007/s11276-018-1887-9

6. Tennakoon, D., Chowdhury, M., \& Luan, T. H. (2018). Cloudbased load balancing using double Q-learning for improved quality of service. Wireless Networks. https://doi.org/10.1007/ s11276-018-1888-8

7. Boulila, W., Al-kmali, M., Farid, M., et al. (2018). A business intelligence based solution to support academic affairs: Case of Taibah University. Wireless Networks. https://doi.org/10.1007/ s11276-018-1880-3 
8. Traore, M., Ndiaye, A., Mbodji, S., et al. (2018). Supervision of a PV system with storage connected to the power line and design of a battery protection system. Wireless Networks. https://doi.org/ 10.1007/s11276-018-1886-x

9. Ellman, J., Lee, N., \& Jin, N. (2018). Cloud computing deployment: A cost-modelling case-study. Wireless Networks. https:// doi.org/10.1007/s11276-018-1881-2

10. Wang, Y., Nörtershäuser, D., Le Masson, S., et al. (2018). Potential effects on server power metering and modeling. Wireless Networks. https://doi.org/10.1007/s11276-018-1882-1

Publisher's Note Springer Nature remains neutral with regard to jurisdictional claims in published maps and institutional affiliations.

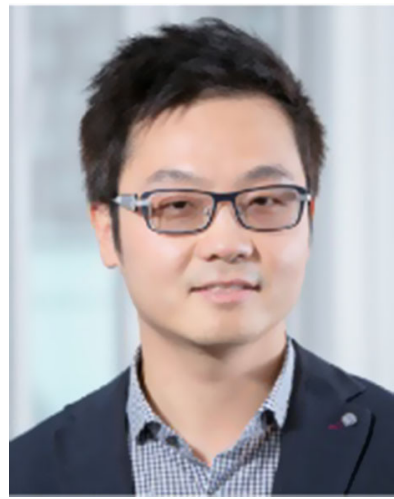

Longzhi Yang is a full professor of Computer Science and Artificial Intelligence, and the Director of Education in the Department of Computer and Information Sciences at Northumbria University in the U.K. He received his Ph.D. degree in computer science from University of Wales, Aberystwyth, U.K. in 2011. His research focuses on $\mathrm{AI}$, data science, automation/control, robotics, computer vision, cybersecurity, additive manufacturing, and other innovative AI applications. Professor Yang is the founding Chair of the IEEE Special Interest Group of Big Data for Cyber Security and Privacy, and the Chair of IEEE Computational Intelligence Society Big Data Task Force. Professor Yang is a Senior Member of IEEE, a professional member of British Computer Society, and a Senior Fellow of Higher Education Academy of U.K.

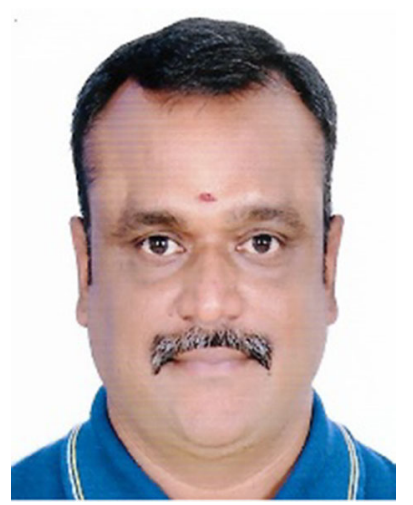

Vijayakumar Varadarajan is currently an Adjunct Professor in School of Computer Science and Engineering, University of New South Wales, Sydney, Australia. He is also a Visiting Postdoc Scientist in Centro de Tecnologia, Federal University of Piauí, Brazil. He was a Professor and Associate Dean for School of Computing Science and Engineering at VIT University, Chennai, India. He has more than 18 years of experience including industrial and institutional. He also served as a Team Lead in industries like Satyam, Mahindra Satyam and Tech Mahindra for several years. He has completed Diploma with First Class Honors. He has completed BE CSE and MBA HRD with First Class. He has also completed ME CSE with First Rank Award. He has completed his PhD from Anna
University in 2012. He has published many articles in national and international level journals/conferences/books. He is a reviewer in IEEE Transactions, Inderscience and Springer Journals. He has initiated a number of international research collaborations with universities in Europe, Australia, Africa, Malaysia, Singapore and North \& South America. He had also initiated joint research collaboration between VIT University and various industries. He is also the Lead Guest Editor for few journals in Inderscience, Springer, Elsevier, IOS, UM and IGI Global. He also organized several international conferences and special sessions in USA, Vietnam, Africa, Malaysia and India including ARCI, IEEE, ACSAT, ISRC, ISBCC, ICBCC etc. His research interests include computational areas covering grid computing, cloud computing, computer networks, cyber security and big data. He received his university-level Best Faculty Award for 2015-2016. He is also a member of several national and international professional bodies including IFSA, EAI, BIS, ISTE, IAENG, CSTA, IEA etc.

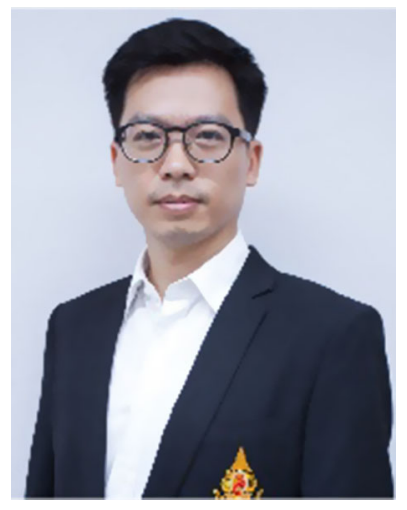

Tossapon Boongoen is an Associate Professor at School of Information Technology, Mae Fah Luang University. $\mathrm{He}$ received his $\mathrm{PhD}$ in Computer Science from Cranfield University, UK in 2003. During 2007-2011, he has been a PDRA and visiting research fellow at Department of Computer Science, Aberystwyth University, UK. He has published articles in well-known venues like IEEE Transactions of Knowledge and Data Engineering, IEEE Transactions of Cybernetics, Machine Learning, Expert Systems with Applications, AI and Law, for instance. In additional, he serves as associate editors of IEEE Access, PeerJ Computer Science, International Journal of AI, and ICT Express. His research interests are AI, machine learning, data science, uncertainty and fuzzy systems.

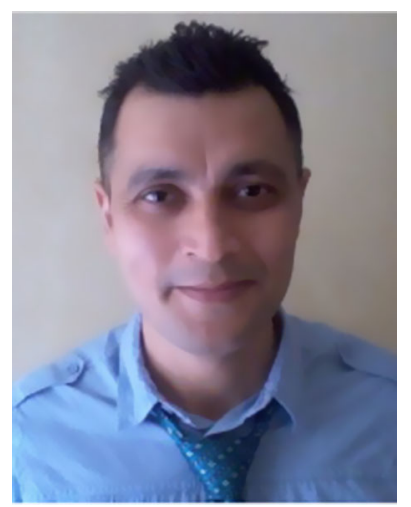

Nitin Naik received the Ph.D. degree in computer science from Aberystwyth University, Aberystwyth, U.K. He additionally holds several academic qualifications: M.Tech., M.Sc., MBA, MSW, B.Sc., and Polytechnic (Electrical Engineering). He has authored more than 100 peer-reviewed papers in the areas of artificial intelligence, cybersecurity, big data, cloud computing, Internet of Things, and game based learning. He is currently a Senior Lecturer with the School of Informatics and Digital Engineering, Aston University, Birmingham, U.K. 